\title{
Innovative Wireless Medium Control Algorithm for Wireless Devices in ISM Bands
}

\author{
Tomas Cuzanauskas \\ Department of Telecommunication \\ Engineering, Vilnius Gediminas \\ Technical University \\ Naugarduko st. 41-211, \\ LT-03227, Vilnius, Lithuania
}

\author{
Aurimas Anskaitis \\ Department of Telecommunication \\ Engineering, Vilnius Gediminas \\ Technical University \\ Naugarduko st. 41-211, LT-03227, \\ Vilnius, Lithuania
}

\begin{abstract}
Since the beginning of the IEEE 802.11 technology its channel access in ISM bands was governed by simple rules to ensure fairness and co-existence: such as upper ceiling on maximum transmitted power, moderate out-of-band emission masks and requirement for tolerance to interference. However, over time these rules became outdated and no longer well suited for current capabilities of devices operating in ISM bands. In this paper we propose a new channel access model for IEEE 802.11 and other devices using unlicensed ISM bands at $2.4 \mathrm{GHz}$ and $5 \mathrm{GHz}$ based on Game Theoretic principles and Cognitive Radio features. It is shown that the proposed channel access method can significantly improve the efficiency of spectrum usage, as well as the quality of service that is experienced by users of ISM bands. Moreover, it would allow abolishing constraint on maximum transmission power and making unnecessary the use of CSMA/CA protocol by replacing it by more advanced Multi-Polling with Game Theory (GT) based protocol.
\end{abstract}

\section{Keywords}

Interference, IEEE 802.11, Game theory, MAC, Cognitive Radio, ISM band.

\section{INTRODUCTION}

The early steps taken by authorities to open $2.4 \mathrm{GHz}$ and 5 $\mathrm{GHz}$ for unlicensed usage has made a worldwide impact to today's wireless devices growth, both in commercial and home premises. In recent years, even higher usage of IEEE 802.11 (Wi-Fi) based devices could have been noticed. As customers' demands are still growing, there are many improvements being introduced of a more advanced version of IEEE 802.11 protocols. However, not much hope was left there for more relaxed transmit power rules or the MAC layer updates, which were still kept the same from the early days and are not up-to-date with current usage trends.

Since the very first days of IEEE 802.11, its operation was based on two key mechanisms for accessing radio channel, namely the DCF (Distributed Coordination Function) and PCF (Point Coordination Function). The DCF mechanism is the only one that has been certified by the Wi-Fi alliance and as of today, it is the access mode of choice implemented in all the Wi-Fi compatible devices. Currently most of the devices rely on IEEE 802.11e and Enhanced Distributed Channel Access (EDCA) mechanism, which additionally adds Quality of Service Support (QoS) for Wi-Fi systems. DCF or EDCA works by employing mechanism called Carrier Sensing Multiple Access/Collision Avoidance (CSMA/CA). The essence of CSMA/CA is that Wi-Fi transceiver senses the channel before transmitting a packet, and if upon transmission it detects that collision did occur (i.e. when two transceivers tried transmitting at the same time), transceiver waits for some time before trying to re-send the packet. This simple mechanism allows fully decentralized and uncoordinated operation of transceivers and therefore was favored by the manufacturers. However, this mechanism exhibits poor performance in highly interfered environments, especially in high density of wireless devices where CSMA/CA decreases the overall performance. Over the time that the Wi-Fi standard has been in place, many attempts have been made to improve the CSMA/CA protocol in a variety of ways $[4,5]$. However, these changes, such as implementing RTS/CTS "handshake" mechanism, do not offer complete solutions [6, 7], whilst proprietary changes to the protocols (outside the IEEE 802.11 standard) can further deteriorate performance [8]. Performance gains are highly dependent on the radio environment and operating in congested environments causes considerable throughput degradation [9]. The latest thinking of Wi-Fi industry may be visible in $802.11 \mathrm{ac}$ as the most recent standard in the IEEE 802.11 family. It has introduced some important improvements to the RTS/CTS mechanism [10] and signal detection threshold [11], but still retained the same dated paradigm of radio channel access.

Set against this background, this paper proposes taking more drastic step towards improving CSMA/CA channel access mechanism. This paper shows that Wi-Fi devices could achieve higher aggregate throughputs, increase the range and quality of the link by allowing the channel access to be governed by Game Theoretic (GT) models. By following a set of simple game rules the quasi-cognitive devices should be perfectly capable of choosing most appropriate transmit power and effecting the packet transmission while seeking the optimum compromise between link range/quality, ambient interference level and its own energy consumption.

The rest of this paper is structured as follows. Section 2 describes the proposed GT-based solution, backed by feasibility analysis and simulations. Section 3 describes the results of practical tests, followed by TCP optimization possibilities in Section 4. Section 5 provides conclusions and ideas for future research.

\section{THE CONCEPT OF POWER ALLOCATION MODEL}

In order to deal with power allocation between devices, game theory approach was selected, which is more suited to control the stochastic environment in ISM band. The proposed framework is used to control the power between connected clients or synchronized Access Points (AP). In proposed framework it is assumed that Access Points are considered as a main entity and transmission power is not limited and is set 
statically or depending on the farthest station's signal level requirements. The framework controls power allocation between active stations in order to contain self-interference. The power allocation is dynamic and highly depends on the environmental conditions. If interference is detected from legacy IEEE 802.11 device, a GT based device tries to increase its signal to noise ratio by increasing transmit power. The stations which are connected to AP are controlled by main entity - agent, which is the Access Point in the framework. AP is the entity, which, depending on the statistical data, makes decisions to transmit power required for each station. By exposing this, we have built a system of quasi-cognitive radios on Wi-Fi physical layer with minor Media Access Control (MAC) layer adjustments. Let's consider a system of $\mathrm{N}$ devices, where $\mathrm{j}$ is considered a player with specific Quality of Service (QoS) requirements. The interference which is created either by other players connected to the same AP or by other legacy devices, is calculated according to the formula [12] below:

$$
\gamma_{j}=\frac{h_{j} p_{j}}{\sum_{i \neq j} h_{i j} p_{i}+n_{0}},
$$

where:

$j$ - a player,

$\gamma_{j}-$ signal Interference Noise Ratio (SINR),

$p_{j}$ - player's $j$ power level in $\mathrm{dBm}$,

$h_{j}$ - antenna gain in $\mathrm{dBi}$,

$p_{i}$ - other players power level in $\mathrm{dBm}$,

$n_{0}$ - environment noise level

The capacity for the given player is defined by Shannon's capacity formula:

$$
C=B \log \left(1+\gamma_{j}\right),
$$

where:

$$
\begin{aligned}
& C-\text { Channel's capacity } \\
& B-\text { Bandwidth } \\
& \gamma_{j}-\text { Signal to Noise Ratio (SNR) }
\end{aligned}
$$

The higher throughput is only reached when the device is operating in near optimal SNR conditions, which in our case should be between 21 and $28 \mathrm{~dB}$. In order to maximize the total capacity of the system we evaluate the utility function, so player $j$ would not increase transmit output power indefinitely:

$$
\begin{aligned}
& \max \sum_{j=1}^{N} u_{j}, \\
& \sum_{i=1}^{N} p_{i} \leq P_{\max },
\end{aligned}
$$

The $P_{\max }$ is used to limit user's power level to prevent the user from increasing power up to a maximum. Then the player's $j$ utility function is defined as [13]:

$$
u_{j}=\log \left(1+\gamma_{j}\right)
$$

The player's output power based on SINR requirements is defined as:

$$
P_{j}=p_{j}\left(1+\frac{S I N R_{\text {target }}-S I N R_{\text {current }}}{S I N R_{\text {target }}}\right),
$$

where:

$P_{j}$ - transmitter power level in $\mathrm{dBm}$, $p_{j}$ - current transmit power level,

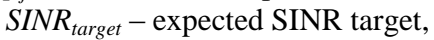
SINR $R_{\text {current }}$ - current device SINR retrieved from device registers.

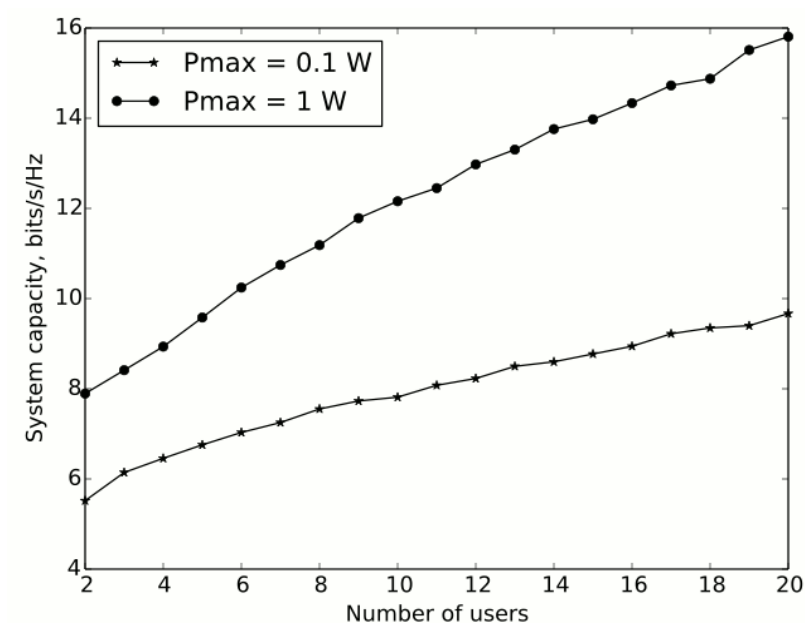

Fig 1: Total system capacity as function of number of users in a 100x100m area. Simulation results of a distributed interference-aware power control game with the $100 \mathrm{~mW}$ limit removed, source code available at [14]

Simulation of the proposed GT framework was modelled by developing Python based software tool, which is available at [14]. The propagation characteristics during simulation are based on [15]. The results of these simulations are presented in Figure 1. From these results, it can be clearly seen that in this scenario, removing the $100 \mathrm{~mW}$ limit and allowing users to operate up to $1 \mathrm{~W}$ EIRP with the proposed channel access and power control algorithm, does have positive consequences on the overall capacity of the links sustained in a given bandwidth as well as better propagation characteristics.

\section{EXPERIMENTAL SETUP AND TESTING}

The main particularities of designing a new Media Access Control (MAC) layer on currently existing of-the-shelf devices is a requirement for compatibility between IEEE 802.11ac and IEEE 802.11n sub-systems. While 802.11n main MAC layer is allocated on the single platform, the 802.11ac uses a split system model, where main MAC state machine code is allocated on offload chipset. The main chain of elements used in IEEE 802.11ac of-the-shelf devices are: Operating System (OS) which handles the application layer specifics for the wireless driver, the host Upper MAC (UMAC) handles all the connection specifics, including the AP state machine. The Target UMAC is mainly used for managing the transmitters and receivers states of the device as well as handling hardware queue and aggregation. This is the part where all the management of the connected stations is performed. Typically in $802.11 \mathrm{n}$ based devices a combination of host and target UMAC is implemented. 
In order to verify and further adjust the theoretical algorithm for practical usage a test setup was built in an outdoor environment consisting of multiple client premises equipment (CPEs) nodes and Access Points (APs). Performance tests of the proposed algorithm was carried out by running comparison against standard $802.11 \mathrm{n}$ protocol with CSMA/CA and as well as Point Coordinated Function (PCF).

Since the proposed algorithm does not incorporate major changes to the IEEE 802.11 PHY characteristics, it is possible to implement the algorithm on off-the-shelf IEEE 802.11n/ac equipment. The proposed power control game model was combined together with our Multi-Polling functionality.

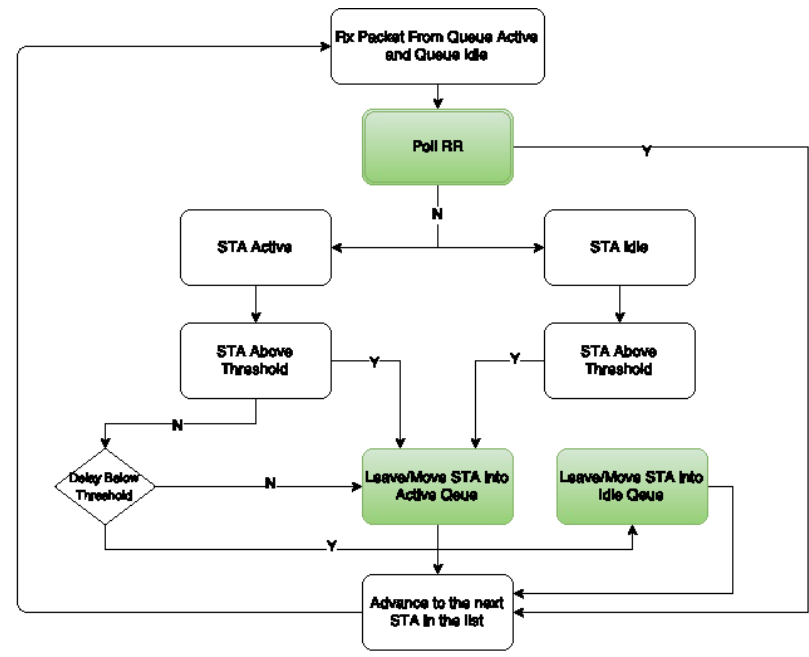

Fig 2: Multi-Polling processing flowchart

The CPEs in multi-polling protocol are split into two groups active and idle. The active CPEs are considered the ones, which generates more throughput than predefined throughput threshold. The idle CPEs are kept in Contention Window (CW) period, where they equally compete for wireless medium. The decision is made based on the following formula:

$$
P_{i}=\frac{\left(N_{i} \cdot A_{i}\right)}{T_{i}}
$$

where:

$N_{i}$ - the number of packets in queue,

$P_{i}-$ the throughput going through station,

$A_{i}$ - the size of the traffic stream from the application,

$T_{i}$-the station's activity period.

From Figure 2, it can be noticed that all CPEs are either polled in Round-Robin method or they are split into two groups. Power allocation is done only for active CPEs devices, while keeping idle CPEs with static power levels. Testing was carried out in an outdoor environment, using Point-to-Multipoint (PtMP) scenario with off-the-shelf $802.11 \mathrm{n} / \mathrm{ac}$ devices. These included $60 \mathrm{CPE}$ devices at $200 \mathrm{~m}$ distance, 23 devices at $5 \mathrm{~km}$ distance and additional 2 devices at $500 \mathrm{~m}$. The AP antenna was 90 degree sector with $18 \mathrm{dBi}$ gain for $\mathrm{AP}$ and $15 \mathrm{dBi}$ gain for CPEs. The signal, depending on the distance, was in the range from $-50 \mathrm{dBm}$ up to -70 $\mathrm{dBm}$. The tests were performed in high interference environment, over city centre. During the testing, the new channel access and power control algorithm worked successfully in establishing and maintaining the links. The output power was self-stabilizing in the range of $25-31 \mathrm{dBm}$. Performance test of the operating links was done using iPerf tool and results are shown in Figure 3. In PtMP scenario the throughput of 802.11n CSMA/CA links decreased due to having multiple streams from different clients, which causes high back-off times as well as hidden node problem and decreases the total throughput. Whereas switching to proposed GT Multi-Polling based algorithm allowed maintaining solid throughput performance.

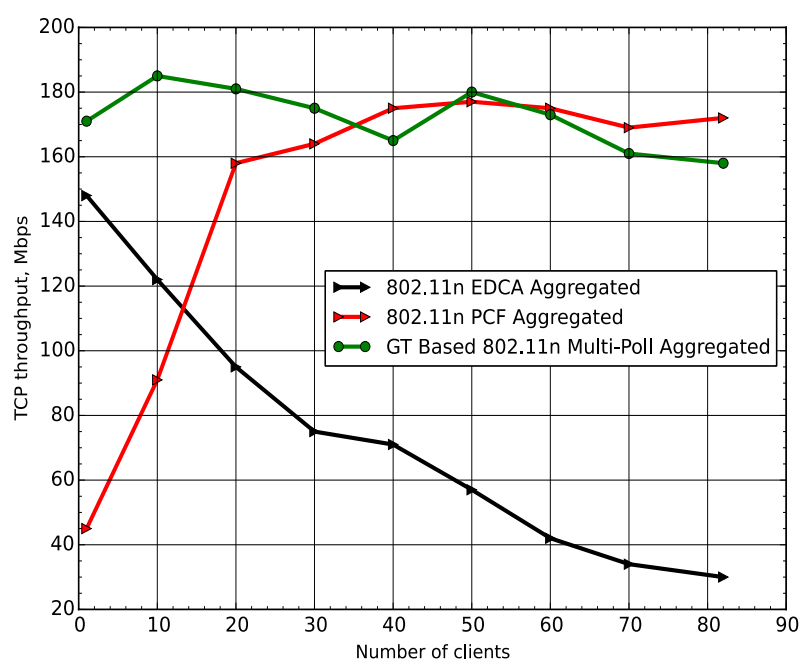

Fig 3: Performance comparison of different protocols

Additionally, a standard Round-Robin (RR) based protocol was tested, which is defined as Point Coordination Function (PCF) according to IEEE 802.11 standard. From results, it can be noticed, that even with standard RR model, it is possible to get good throughput figures when there are more active clients. However, wireless medium is wasted for management frames, when there are only few active clients out of 82 connected. This is not seen on GT Based Multi-Polling protocol, which maintains the throughput in both low activity and high activity conditions. Results confirm that CSMA/CA has severe limitations when operating in dense environments and its limitation can be effectively overcome by utilizing the proposed GT-based channel access and power control algorithm.

Furthermore, the reason for such a low IEEE 802.11 EDCA performance was investigated. While it seems that Downlink Capacity when all CPEs are just receiving data is high, this is not the case when the CPEs are sending more data. The Figure 4 displays the Downlink Capacity when number of active CPEs increases. This might be a result of both fairness problematic and hidden node issue when network has more active CPEs. However, both PCF and GT Based Multi-Polling perform quite well and Downlink capacity is not decreased when amount of active clients had risen. This allows concluding, that without power limitations and improved MAC layer it is possible to handle far higher number of stations and at the same time increase spectrum efficiency. 


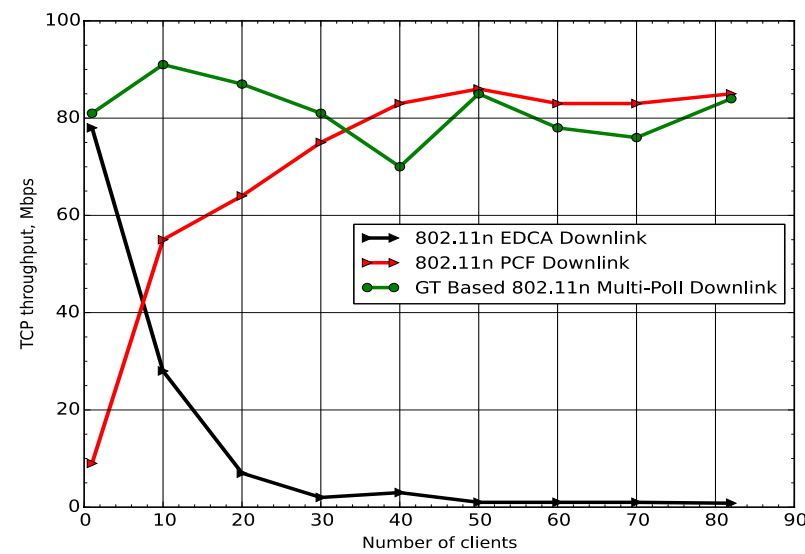

Fig 4: Downlink performance comparison of different protocols

In the last test a measurement of total system capacity with the latest IEEE 802.11ac standard was performed. The same GT protocol was implemented on IEEE 802.11ac of-the-shelf hardware and tested on $200 \mathrm{~m}$ and $500 \mathrm{~m}$ distance. The results in the Figure 5 display aggregated throughput GT Based solution versus standard IEEE 802.11 EDCA mechanism. The GT based protocol throughput is quite significant, mainly due to Multi-Poll solution which handles wireless medium more efficiently and decreases collisions that usually occur due to hidden node problem on IEEE 802.11 EDCA mechanism.

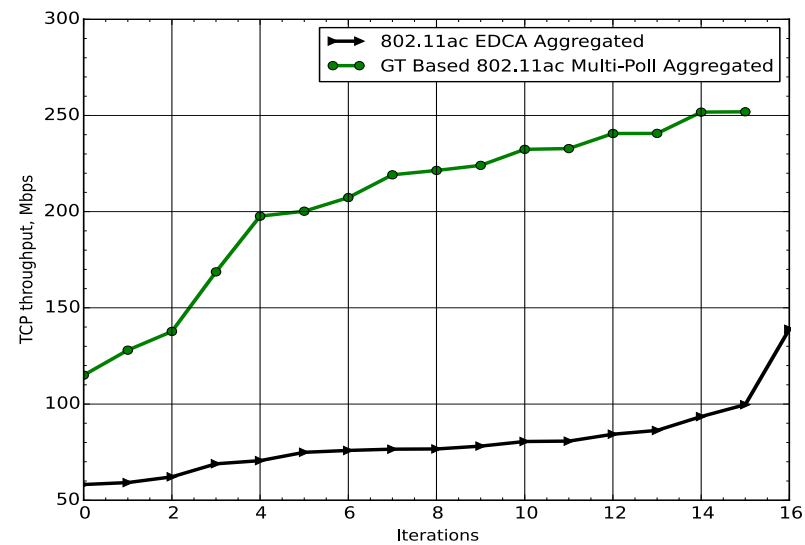

Fig 5: Performance comparison of IEEE 802.11ac with GT and with standard EDCA wireless medium access mechanisms

\section{TCP OPTIMIZASTION IN GT PROTOCOL}

Due to dynamics of wireless networks, the Downlink and Uplink capacity might vary. This is called an asymmetrical link situation, where either Uplink or Downlink is limited due to interference or other situations. To deal with such cases, a new TCP ACKs filtering technique was implemented, which is mainly adopted in satellite communications and in other type of asymmetrical networks. The TCP ACKs flow guarantees growing throughput and TCP mechanism requires a steady and stable flow of ACKs. While usually, this is not a problem in wired communication in wireless networks we might have a case, where TCP ACKs are lost or outdated due to wireless medium occupation.

TCP accelerator mechanism is implemented both in CPEs and Ap. The TCP ACKs are moved to different queue than all the data packets. When the CPEs receive token only the up-to- date ACKs are sent firstly from TCP ACKs queue. All the outdated or redundant ACKs, which acknowledge the same data frames, are dropped. When CPEs receive permission for transmission, firstly the TCP ACKs queue is sent and later a data queue. This allows handling jitter and latency, which is one of the important parameters for steady TCP flow. During testing on our IEEE 802.11ac devices we have relied on standard CUBIC TCP implementation [27], which is common between Linux based systems. The CUBIC TCP provides us one important capability and allows not to rely on TCP ACKs to increase the TCP Window size. This allows removing as many redundant TCP ACKs as possible, thus increasing the spectral efficiency.

In the first test a simplex traffic was generated by using iPerf tool. While simplex traffic was running a flow of TCP ACKs was measured on the device. The results are displayed in Figure 6, as it was noticed TCP ACKs creates almost steady 2 Mbps traffic from CPE to AP. When TCP filtering technique is enabled, the number of TCP ACKs decreases and less than $0.25 \mathrm{Mbps}$ is generated from CPE to AP. This decreases redundant TCP ACKs and allows using wireless medium more efficiently.

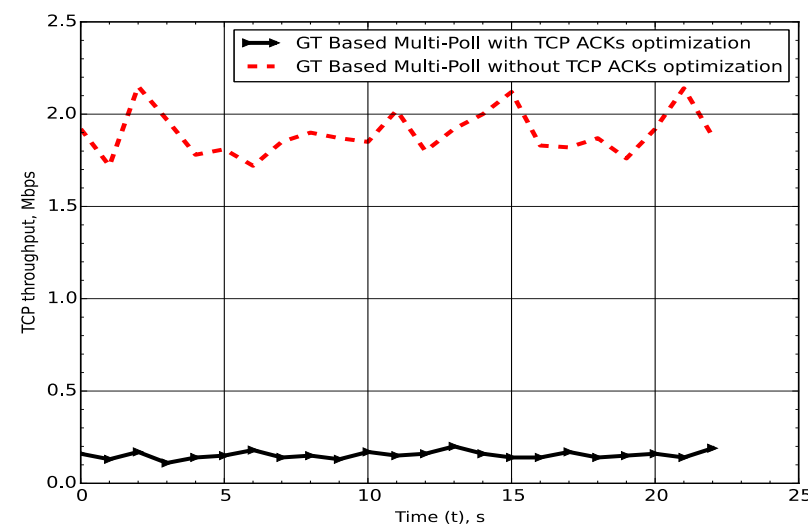

Fig 6: TCP ACKs generated throughput with and without TCP ACK acceleration

In the last test GT Based solution was compared with and without TCP filtering technique. From Figure 7 it can be noticed that TCP ACKs filtering allows increasing the overall throughput up to $10 \%$ in different time periods. The fluctuations which are seen from the figure are caused by the interference of already deployed nearby wireless devices.

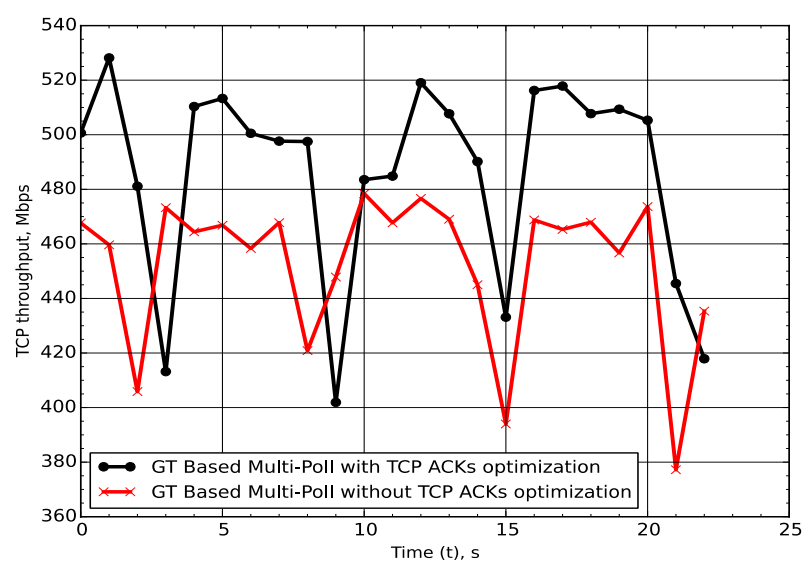

Fig: 7: Throughput with TCP ACKs acceleration on GT solution 


\section{SUMMARY AND CONCLUSIONS}

This paper proposed a novel GT-based algorithm for channel access and power control for possible evolution of IEEE 802.11 and other wireless devices using ISM bands. Reported simulations and practical experiments confirm the practical feasibility of the algorithm and its positive effect on increasing of efficient utilization of subject band.

It is especially notable that the proposed algorithm allowed achieving higher throughput compared to standard CSMA/CA or PCF approaches. This suggests that the proposed intelligent interference-aware autonomous power coordination mechanism together with Multi-Poll MAC adaption may be even used as a substitute for CSMA/CA. Additionally, TCP sessions handling optimization shows up to $10 \%$ higher throughput with high density of connected nodes.

Furthermore, it is noticed that current strict power limits might be relaxed for the maximum transmit power in certain scenarios. This would provide better Quality of Experience (QoE) for end customers as well as improve connectivity in outdoor and indoor deployments. The current MAC layer mechanisms, by using our proposed combinations, might allow more efficient spectrum usage in PtMP deployments, while power allocation mechanism would provide benefit for current and new wireless applications in unlicensed ISM band.

\section{REFERENCES}

[1] J. Scott Marcus, John Burns. 2013. Study on Impact of traffic off-loading and related technological trends on the demand for wireless broadband spectrum. Report for the European Commission, 2013. Available: http://dx.doi.org/10.2759/19531

[2] E. Haghani, M. Krishnan and A. Zakhor, "Adaptive Carrier Sensing for Throughput Improvement in IEEE 802.11 Networks", Proceedings of the IEEE Globecom 2010, Dec 2010, pp. 1-6.

[3] Q. Shen, X. Fang, R. Huang, P. Li, and Y. Fang, "Improving Throughput by tuning carrier sensing in 802.11 wireless networks," Journal Computer Communications, vol. 32 Issue 11, pp. 1263-1270, July 2009.

[4] P. Chatzimisios, A. Boucouvalas, and V. Vitsas, "Effectiveness of RTS/CTS handshake in 802.11a Wireless LANs," IEEE 2004 Electronics Letters Online Available http://dx.doi.org/10.1049/el:20040510

[5] I. Tinnirello, D. Giustiniano, L. Scalia and G. Bianchi, "On the side-effects of proprietary solutions for fading and interference mitigation in IEEE $802.11 \mathrm{~b} / \mathrm{g}$ outdoor links," Computer Networks Volume 53, Issue 2, pp 141152, Feb 2009.
[6] A. Jardosh, K. Ramachandran, K. Almeroth and E. Belding-Royer, "Understanding Congestion in IEEE $802.11 \mathrm{~b}$ Wireless Networks," Proceedings of the 5th USENIX conference on Internet Measurement IMC, 2005, pp. 25-25.

[7] R. Chandra, R. Mahajan, T. Moscibroda, R. Raghavendra, and P. Bahl, "A case for adapting channel width in wireless networks,". In ACM SIGCOMM Computer Communication Review, vol. 38, no. 4, pp. 135-146, August 2008.

[8] Gast and Matthew, 802.11 ac: A Survival Guide. O'Reilly Media, 2013.

[9] S. Pediaditaki, M. Marina, and D. Tyrode, "Trafficaware channel width adaptation in long-distance 802.11 mesh networks," in Proceedings of the 15th ACM international conference on Modeling, analysis and simulation of wireless and mobile systems ACM, October 2012, pp. 261-270.

[10] B. Wang, Y. Wu, and K. J. R. Liu, "Game theory for cognitive radio networks: an overview," Computer Networks, The Int. J. of Computer and Telecommunications Networking, vol. 54, no. 14, pp. 2537-2561, 2010

[11] R. Etkin, A. Parekh and D. Tse, "Spectrum sharing for unlicensed bands," IEEE J. Select. Areas Comm., vol. 25, 2007, pp.517-528.

[12] Yin Zhiming and Xie. Jianying, "Joint power and rate allocation for the downlink in wireless CDMA data networks," in Proceedings of 14th IEEE 2003 International Symposium on Personal, Indoor and Mobile Radio Communication, Beijing, 2003. Available http://dx.doi.org/10.1109/PIMRC.2003.1264287

[13] R. Kazemi, R. Vesilo, E. Dutkiewicz, and Liu. Ren Liu, "Dynamic power control in wireless body area networks using reinforcement learning with approximation," in Proceedings of Personal Indoor and Mobile Radio Communications (PIMRC) IEEE 22nd International Symposium, Canada, September 2011. Available http://dx.doi.org/10.1109/PIMRC.2011.6139908

[14] Software for simulation of channel access and power control game. Available: http://www.cost-terra.org/ourpublications

[15] Propagation data and prediction methods for the planning of indoor radio communication systems and the radio local area networks in the frequency range $900 \mathrm{MHz}$ to $100 \mathrm{GHz}$, ITU-R Recommendation P.1238, ITU, Geneva, 2001. 